\title{
Rastreamento cognitivo e sintomas depressivos em idosos iniciantes em programa de exercício físico
}

Cognitive tracking and depressive symptoms in elderly

starting out on physical exercise program

Lucélia Justino Borges', Tânia R. Bertoldo Benedetti', Giovana Zarpellon Mazo²

\section{RESUMO}

Objetivo: Identificar o estado de saúde mental de idosos iniciantes em um programa de exercício físico e analisar a associação entre os indicadores sociodemográficos com a pontuação das escalas geriátricas. Métodos: Participaram do estudo 121 idosos, com idade média de 68,69 $\pm 6,46$ anos, cadastrados nos Centros de Saúde de Florianópolis/SC. Aplicou-se o Mini-Exame do Estado Mental (MEEM) e a escala de depressão geriátrica (GDS). Resultados: a prevalência de transtornos cognitivos foi de 9,1\% e de depressão de 17,4\%. Tanto a escolaridade quanto a renda apresentaram associação estatística significativas com a pontuação do MEEM ( $p<0,001$ e $p<0,001$, respectivamente). Nenhuma das variáveis estudadas (estado civil, idade e renda) associou-se com a pontuação da GDS ( $p=0,986, p=0,226$, $p=0,302)$, respectivamente. Observou-se relação inversa e significativa entre MEEM e GDS $(r=-0,201 ; p=0,02)$. Conclusões: Evidenciaram-se prevalências significativas para depressão e transtornos cognitivos em idosos iniciantes em um programa de exercício físico. Para esta população, quanto maior a escolaridade e a renda, melhor é o desempenho no MEEM. Detectou-se, ainda, que os indivíduos que apresentaram sintomas depressivos obtiveram pontuação abaixo do normal no MEEM.

\section{ABSTRACT}

Objective: To identify the mental health status of elderly people starting out on an exercise program and to analyze the relationship between socio-demographic and economic indicators and scores on geriatric assessment scales. Methods: The study enrolled 121 elderly people, with a mean age of 68.69 \pm 6.46 years, registered at Health Centers in Florianopolis. The Mini-Mental State Examination (MMSE) and the Geriatric Depression Scale (GDS) were applied to these subjects. Results: The prevalence of cognition disorders was $9.1 \%$ and the prevalence of depression was $17.4 \%$. Both educational level and income exhibited statistically significant associations with MMSE scores ( $p<0.001$ and $p<0.001$, respectively). None of the variables studied (marital status, age and income) were associated with $\operatorname{GDS}$ scores $(p=0.986, p=0.226, p=0.302)$, respectively.

1 Programa de Pós-Graduação em Educação Física da Universidade Federal de Santa Catarina (UFSC) - Bolsista Capes. 2 Programa de Pós-Graduação em Ciência do Movimento Humano da Universidade do Estado de Santa Catarina (Udesc). 


\section{Key-words}

Elderly, depression, cognition disorders, exercise.
A significant inverse relationship was observed between MMSE and GDS scores ( $r=-0.201$; $p=0.02)$. Conclusions: There were significant prevalence rates of both depression and cognitive disorders among elderly people beginning a physical exercise program. In this population, the higher the educational level and income, the better the performance on the MMSE scale. It was also detected that individuals who exhibited depressive symptoms scored lower than normal on the MMSE.

\section{INTRODUÇÃO}

O processo de envelhecimento populacional tem sido acompanhado com o aumento das doenças psiquiátricas, entre as quais a demência e a depressão se destacam como os transtornos mentais mais comuns em idosos (Reys et al., 2006). A demência afeta cerca de 5\% dos idosos com 65 anos de idade e $20 \%$ com 80 anos ou mais de idade (Jorm e Jolley, 1998; Herrera et al., 2002) e se apresenta como uma das maiores causas de morbidade entre idosos. A depressão tem taxas de prevalência entre 5\% e 35\%, variando de acordo com o nível de gravidade (Almeida et al., 1998), porquanto a prevalência de sintomas depressivos na população mundial acima de 65 anos de idade, vivendo na comunidade, varia entre 10,3\% e 13,5\% (Snowdon, 2002) e no Brasil chega a 14,3\% (Blay et al., 1999).

Mesmo sendo uma doença psiquiátrica muito comum entre os idosos, a depressão freqüentemente não é diagnosticada, e o acometido não recebe tratamento (Oliveira et al., 2006). Parte das dificuldades encontradas no diagnóstico se deve ao fato de que essa doença é uma síndrome heterogênea tanto quanto a etiologia quanto à resposta ao tratamento (Ávila e Botino, 2006). Outro aspecto apontado é que os idosos, com maior freqüência de depressão apresentam quadros atípicos ou particularidades, como problemas clínicos e sociais simultâneos, que podem dificultar o diagnóstico (Stoppe Júnior e Louzã Neto, 1998). Portanto, é fundamental que os profissionais da saúde tenham familiaridade com as características da depressão no idoso e estejam preparados para investigar a presença de seus sintomas. Nesse contexto, o uso sistemático de escalas de depressão pode facilitar a detecção desses casos, auxiliando a prática clínica e intervindo positivamente (Almeida e Almeida, 1999).

O uso de escalas de rastreamento de demência também pode facilitar a intervenção adequada (Alexopoulos et al., 1993). Apesar de ainda gerar controvérsias, o diagnóstico precoce das demências possibilita intervenção terapêutica, diminui os níveis de estresse para os familiares, reduz riscos de acidentes, prolonga a autonomia e, em alguns casos, evita ou retarda o início do processo demencial (Petersen et al., 2001).
Assim sendo, destaca-se a importância da utilização sistemática de escalas geriátricas de saúde mental e o reconhecimento das variáveis que possam influenciar o desempenho do indivíduo. Assim sendo, reconhece-se a influência da escolaridade e da idade no desempenho dos testes de rastreio para a demência, especificamente, o Mini-Exame do Estado Mental (MEEM), que é o teste mais utilizado no mundo (Lourenço e Veras, 2006). Vários estudos em diferentes países demonstraram que, mesmo em pessoas que não apresentaram evidências de déficit cognitivo, quanto menor a escolaridade e maior a idade, menor era a pontuação no MEEM (Bertolucci et al., 1994; Diniz et al., 2007).

Diante disso, em países desenvolvidos, nos quais a escolaridade dos idosos é elevada, ultrapassando 12 anos de escolaridade formal, o ponto de corte comumente usado em estudos clínicos e epidemiológicos para a demência é de 24 pontos (Fratiglioni et al., 1993). Nos países em desenvolvimento, como o Brasil, em virtude do grande número de indivíduos analfabetos e com baixa escolaridade, a estratificação dos pontos de corte de acordo com a escolaridade assume grande importância. Assim, desde a versão do MEEM publicada para a população brasileira (Bertolucci et al., 1994), foi observada clara e forte influência do nível de escolaridade sobre os escores totais do MEEM, fato confirmado por demais estudos (Almeida, 1998; Brucki et al., 2003; Diniz et al., 2007).

Em relação à depressão, os estudos apresentam entre as principais características associadas, as variáveis demográficas, como idade avançada, ser do sexo feminino (Cacciatore, 1998) e estado civil (Camarano, 1999), além das condições de saúde, como o declínio funcional, doenças crônicas e prejuízo cognitivo (Penninx et al., 2000) e as condições sociais precárias (Camarano, 1999).

Buscando analisar as principais características associadas à depressão e à demência, a literatura apresenta pesquisas, envolvendo idosos atendidos em ambulatório, idosos vivendo na comunidade ou institucionalizados (Lourenço e Veras, 2006; Diniz et al., 2007; Leite et al., 2006; Oliveira et al., 2006). No entanto, evidencia-se número reduzido de estudos envolvendo a avaliação do estado mental e associações entre variáveis sociodemográficas em idosos praticantes de atividade física. 
Levando em consideração os aspectos abordados, foi proposto o projeto de pesquisa, enfocando saúde mental e prática de exercício físico em Centros de Saúde de Florianópolis. Para tanto, o presente estudo encontra-se vinculado à primeira etapa do projeto, sendo delineado os seguintes objetivos: (1) identificar o estado de saúde mental (sintomas depressivos e transtornos cognitivos) de idosos iniciantes em um programa de exercício físico nos Centros de Saúde de Florianópolis; (2) analisar a associação entre os indicadores sociodemográficos (escolaridade, faixa etária, renda, estado civil) com a pontuação das escalas geriátricas (MEEM e escala de depressão geriátrica).

\section{MÉTODO}

O estudo obteve aprovação do Comitê de Ética em Pesquisa da Universidade Federal de Santa Catarina (UFSC) (nº 011/06). Todos os indivíduos após o esclarecimento sobre o estudo aceitaram participar da pesquisa, assinando o Termo de Consentimento Livre e Esclarecido (TCLE).

\section{População e amostra}

O programa de exercício físico implantado nos centros de saúde (CS) é uma das ações norteadoras do "Capital Idoso", lançado em outubro de 2006. Foi idealizado pela Secretaria de Saúde do Município de Florianópolis, tendo como parceiras as UFSC e Universidade do Estado de Santa Catarina (Udesc), para atender idosos portadores de doenças crônicas, cadastrados nos CS da sua comunidade. Acrescenta-se que esse programa contempla diversas áreas profissionais da saúde: educação física, nutrição, enfermagem, medicina, odontologia, entre outras. Assim, dentro de cada área profissional, estão sendo desenvolvidas propostas de intervenção e estudos voltados aos idosos. Especificamente, na área de abrangência da educação física, o programa denominado "Floripa Ativa" tem por objetivo desenvolver e manter a capacidade funcional e a saúde mental dos idosos, por meio da implantação de um programa de ginástica desenvolvido nos CS. O programa é oferecido três vezes por semana, com duração de 60 minutos por sessão. As atividades do programa iniciaram em junho de 2006, como um projeto-piloto, atendendo quatro CS de Florianópolis que são: Córrego Grande, Rio Tavares, Saco Grande e Policlínica do Estreito.

Entre os meses de junho de 2006 e março de 2007, 122 idosos (18 do sexo masculino e 104 do feminino) encontravam-se cadastrados e participavam regularmente do "Floripa Ativa", sendo essa a população-alvo desse estudo. Para tanto, houve a exclusão de um sujeito por apresentar incapacidade para responder o questionário em forma de entrevista, pois era surdo e não se comunicava nem pela linguagem brasileira dos sinais, nem por leitura labial. Dessa maneira, a amostra do presente estudo foi composta por
121 idosos (104 mulheres e 17 homens), com idade entre 60 a 86 anos.

\section{Instrumentos e coleta de dados}

Os instrumentos aplicados foram: MEEM versão brasileira, traduzido e adaptado por Bertolucci et al. (1994); GDS, versão abreviada com 15 perguntas (Yesavage et al., 1983) e informações sociodemográficas (idade, sexo, estado civil, renda e escolaridade) obtidas por meio das fichas de cadastro do programa.

Utilizou-se categorização/classificação para as variáveis sociodemográficas estudadas. Para a idade, os sujeitos foram divididos em três faixas: 60 a 69, 70 a 79 e mais de 80 anos, porquanto, para obtenção da idade, levou-se em consideração a data registrada no documento de identidade. Quanto ao estado civil classificou-se em três categorias: casado, viúvo e agrupou-se em uma única categoria: separados, solteiros e divorciados. O referencial utilizado para definir a renda familiar mensal dos sujeitos foi o número de salários mínimos, conforme metodologia utilizada pelo Instituto Brasileiro de Geografia e Estatística (IBGE) na Pesquisa de Orçamento Familiar (POF) de 2002/2003. Na data da aplicação do questionário (julho/2006 a março/2007), o valor do salário mínimo vigente era de $\mathrm{R} \$ 350,00$ (US\$ 184.21). Tendo como base o estudo do Rio de Janeiro (Veras et al., 2007), que utilizou como renda familiar mensal o resultado de toda a receita angariada pela unidade familiar: salários, pensões, aposentadorias, contribuições de outros familiares e amigos, ajudas de custo, renda proveniente de propriedades, entre outros. Com base em estudos recentes publicados (Lourenço e Veras, 2006; Veras et al., 2007), a renda em salários foi distribuída em cinco categorias: até 1,9; de 2 a 3,9; de 4 a 5,9; de 6 a 9,9 e mais de 10 salários. Ressaltam-se que para a análise estatística utilizaram-se quatro categorias, sendo agrupadas as duas últimas descritas anteriormente, ficando a última categoria "seis ou mais salários". Em relação à escolaridade, adotou-se a classificação por anos de estudo formal: analfabetos, de 1 a 3 anos, de 4 a 7 anos e mais de 8 anos (Bertolucci et al., 1994).

A coleta de dados foi realizada no período compreendido entre julho de 2006 e março de 2007, sendo aplicadas entrevistas individuais àqueles idosos que aderiam ao programa "Floripa Ativa". As entrevistas foram realizadas no próprio local onde são realizadas as aulas, em horários divergentes a elas, sendo agendados horários antes e após as aulas. A equipe de coleta de dados foi composta pela autora principal deste artigo e por dois acadêmicos, que foram devidamente treinados para aplicação dos instrumentos.

\section{Análise estatística}

Os dados foram analisados por meio do pacote estatístico SPSS para Windows, versão 11.0. Foram utilizadas as análises estatísticas descritivas para a elaboração do perfil sociode- 
mográfico da amostra e para classificação percentual do MEEM e da GDS. O teste não paramétrico de Kruskall-Wallis foi utilizado para verificar a diferença entre a pontuação do MEEM e as variáveis sociodemográficas (escolaridade, faixa etária e renda), bem como para avaliar a pontuação da GDS com as variáveis sociodemográficas (estado civil, renda e escolaridade). A correlação de Spearman foi usada para avaliar a associação entre pontuações do MEEM e da GDS. O nível de significância de $\mathrm{p}<0,05$ foi adotado para todas as análises.

\section{RESULTADOS}

Dos 121 indivíduos avaliados, 86\% eram mulheres. A idade média foi de 68,69 $\pm 6,46$ anos. Quanto à escolaridade, $9 \%$ eram analfabetos e 43,8\% tinham de quatro a sete anos de estudo. Quanto ao estado civil 56,2\% eram casados; 41,1\% dos indivíduos recebiam de 2 a 3,9 salários mínimos (Tabela 1), visto que a renda familiar variou de $\mathrm{R} \$ 350,00$ (um salário mínimo) a R\$ 7.700 (vinte e dois salários mínimos).

Tabela 1. Características sociodemográficas dos idosos iniciantes em um programa de exercício físico nos CS de Florianópolis $(n=121)$

\begin{tabular}{|c|c|c|}
\hline & Freqüência & $\%$ \\
\hline \multicolumn{3}{|l|}{ Sexo } \\
\hline Masculino & 17 & 14 \\
\hline Feminino & 104 & 86 \\
\hline \multicolumn{3}{|l|}{ Idade } \\
\hline $60-69$ anos & 66 & 54,5 \\
\hline 60-79 anos & 46 & 38 \\
\hline 80 anos ou mais & 09 & 7,5 \\
\hline \multicolumn{3}{|l|}{ Escolaridade (anos de estudo formal) } \\
\hline Analfabeto & 09 & 7,4 \\
\hline $1-3$ anos & 40 & 33,1 \\
\hline 4-7 anos & 43 & 43,8 \\
\hline 8 anos ou mais & 19 & 15,7 \\
\hline \multicolumn{3}{|l|}{ Estado civil } \\
\hline Casado (a) & 68 & 56,2 \\
\hline Viúvo (a) & 43 & 35,5 \\
\hline Separado(a); solteiro(a); divorciado(a) & 10 & 8,3 \\
\hline \multicolumn{3}{|l|}{ Renda* $\left(S M^{* *}\right)$} \\
\hline Até 1,9 & 23 & 21,5 \\
\hline 2 a 3,9 & 44 & 41,1 \\
\hline 4 a 5,9 & 22 & 20,6 \\
\hline 6 a 9,9 & 12 & 11,2 \\
\hline 10 ou mais & 06 & 5,6 \\
\hline
\end{tabular}

${ }^{*} \mathrm{n}=107$ (14 casos não declarados); **SM = salário mínimo; valor = R\$350,00 (US\$ 179.48).

A prevalência de transtornos cognitivos foi de 9,1\%, porquanto, entre os locais pesquisados, o CS do Córrego Grande destacou-se com 45,4\% do percentual obtido. A Tabela 2 apresenta a pontuação no MEEM geral e pela escolaridade. Verificou-se que as medianas do MEEM por classificações de renda e escolaridade apresentaram diferença estatística, em contrapartida, essa diferença não foi observada entre as medianas da faixa etária (Tabela 3).
Tabela 2. Pontuação do MEEM global e por escolaridade (anos de estudo formal) de idosos iniciantes em um programa de exercício físico nos CS de Florianópolis $(n=121)$

\begin{tabular}{lccccc}
\hline & Global & Analfabetos & $1-3$ anos & $4-7$ anos & $\begin{array}{c}8 \text { anos } \\
\text { ou mais }\end{array}$ \\
\hline Média & 25,64 & 20,56 & 24,28 & 26,77 & 27,74 \\
(DP) & 3,24 & 2,55 & 2,79 & 2,71 & 1,82 \\
Mediana & & 20 & 24 & 28 & 28 \\
$\begin{array}{l}\text { Pontuação } \\
\text { referência }\end{array}$ & Bertolucci et al. & 18 & 21 & 24 & 26 \\
\hline
\end{tabular}

Tabela 3. Mediana do MEEM por renda, faixa etária e escolaridade (anos de estudo formal) de idosos iniciantes em um programa de exercício físico nos CS de Florianópolis $(n=121)$

\begin{tabular}{|c|c|c|c|}
\hline & Freqüência & Mediana ${ }^{* * *}$ & Kruskall-Wallis \\
\hline Renda* & & & $<0,001$ \\
\hline Até $1,9 \mathrm{SM}^{* *}$ & 23 & $25^{\mathrm{A}}$ & \\
\hline $2-3,9 \mathrm{SM}$ & 44 & $25^{\mathrm{A}}$ & \\
\hline $4-5,9 \mathrm{SM}$ & 22 & $28^{B}$ & \\
\hline $6 \mathrm{SM}$ ou mais & 18 & $28^{B}$ & \\
\hline Faixa Etária & & & 0,087 \\
\hline 60-69 anos & 66 & $26,5^{A}$ & \\
\hline $70-79$ anos & 46 & $26^{A}$ & \\
\hline 80 anos ou mais & 09 & $24^{A}$ & \\
\hline Escolaridade & & & $<0,001$ \\
\hline Analfabetos & 09 & $20^{A}$ & \\
\hline $1-3$ anos & 40 & $24^{B}$ & \\
\hline 4-7 anos & 43 & $28^{c}$ & \\
\hline 8 anos ou mais & 19 & $28^{c}$ & \\
\hline
\end{tabular}

* $(\mathrm{n}=107) ;{ }^{* *} \mathrm{SM}=$ salário mínimo; ${ }^{* * *}$ Medianas seguidas por letras iguais não diferem pelo teste de MannWhitney $(p>0,05)$.

De acordo com os resultados da pontuação da GDS, 17,4\% apresentaram suspeita à depressão, alcançando seis pontos ou mais. Ao verificar essa prevalência entre os locais pesquisados, o CS do Rio Tavares apresentou maior percentual (33,4\%), seguido do CS Córrego Grande (23,8\%), Saco Grande $(23,8)$ e Policlínica do Estreito, com 19\%. As medianas da GDS por classificações de renda, faixa etária e estado civil não apresentaram diferença estatística significativas (Tabela 4).

Tabela 4. Mediana da GDS por renda, faixa etária e estado civil de idosos iniciantes em um programa de exercício físico nos CS de Florianópolis $(n=121)$

\begin{tabular}{lccc}
\hline & Freqüência & Mediana** $^{* *}$ & Kruskall-Wallis \\
\hline Renda* & 23 & $4^{\mathrm{A}}$ & 0,302 \\
Até 1,9 SM** & 44 & $3^{\mathrm{A}}$ & \\
$2-3,9$ SM & 22 & $2,5^{\mathrm{A}}$ & \\
$4-5,9$ SM & 18 & $2^{\mathrm{A}}$ & \\
6 SM ou mais & & & 0,226 \\
Faixa Etária & 66 & $3^{\mathrm{A}}$ & \\
$60-69$ anos & 46 & $3^{\mathrm{A}}$ & \\
$70-79$ anos & 09 & $4^{\mathrm{A}}$ & \\
80 anos ou mais & & & 0,986 \\
Estado Civil & 68 & $3^{\mathrm{A}}$ & \\
Casado & 43 & $3^{\mathrm{A}}$ & \\
Viúvo & 10 & $3^{\mathrm{A}}$ & \\
Separado; solteiro; divorciado & & & \\
\hline
\end{tabular}

$*(n=107) ;{ }^{*}$ SM $=$ salário mínimo; ***Medianas seguidas por letras iguais não diferem pelo Teste de MannWhitney $(p>0,05)$. 
Analisando a freqüência entre presença e ausência de sintomas depressivos e a classificação do MEEM, foram observadas $80,2 \%$ de concordância entre as classificações. Pela correlação de Spearman detectou-se que existe relação inversa entre MEEM e GDS (Tabela 5).

Tabela 5. Freqüência entre presença ou ausência de sintomas depressivos e a classificação do MEEM. Associação entre as pontuações do MEEM e da GDS de idosos iniciantes em um programa de exercício físico nos CS de Florianópolis $(n=121)$

\begin{tabular}{lccc}
\hline & \multicolumn{2}{c}{ MEEM } & Correlação de \\
\cline { 2 - 3 } & Abaixo & Normal & Spearman \\
\hline Presença de sintomas depressivos & 04 & 17 & \\
Ausência de sintomas depressivos & 07 & 93 & \\
Pontuação MEEM $\times$ GDS & & & $-0,201^{*}$ \\
\hline
\end{tabular}

*significativo $(p<0,05)$ pelo teste $t$ de Student.

\section{DISCUSSÃO}

Verificou-se que a prevalência de transtornos cognitivos foi de 9,1\%. Quando analisados os escores negativos para o MEEM, constatou-se que o maior percentual de pontuação abaixo do normal $(54,5 \%)$ foi encontrado no grupo de escolaridade de 4 a 7 anos, fato que pode ser explicado pelo maior número de sujeitos nessa categoria. Embora os parâmetros estabelecidos pelos pontos de corte aqui utilizados devam ser vistos com cautela, uma vez que outros índices já foram sugeridos por pesquisas com a população brasileira (Brucki et al., 2003; Almeida, 1998). Os achados neste estudo parecem sugerir que o percentual de participantes com transtornos cognitivos é menor do que os encontrados em estudos epidemiológicos, como a pesquisa de Ishizaki et al. (1998) que encontrou 21,8\% dos idosos de uma cidade do norte do Japão com indicadores de demência. No Brasil, os resultados encontrados também diferem dos demais. A pesquisa preliminar do rastreamento cognitivo da população idosa, em uma cidade do Sudeste, apontou prevalência de transtornos cognitivos de 16,5\% (Laks et al., 2003).

Entre as diferenças sociodemográficas significativas encontradas entre os indivíduos demenciados e aqueles cognitivamente preservados estão as variáveis: faixa etária, escolaridade e renda. Assim sendo, verificou-se no presente estudo que tanto a escolaridade quanto a renda influenciaram o desempenho no teste, ou seja, quanto maior a escolaridade e a renda, melhor o desempenho do MEEM.

Em relação à escolaridade, estes resultados estão em ampla concordância com a literatura (Bertolucci et al., 1994; Almeida, 1998; Laks et al., 2003; Diniz et al., 2007). Em um estudo longitudinal com idosos de 80 anos de idade ou mais realizado, no sul do país, verificou-se que o desempenho no MEEM apresentou um efeito principal para escolaridade $(F(1,36)=7,213, p<0,05)$. Os idosos com menos anos de es- colaridade tiveram resultado significativamente inferior no MEEM ( $M=18,81$; $D P=0,85)$, quando comparados com idosos com mais anos de escolaridade $(M=22,34$; $D P=0,99)$ (Argimon e Stein, 2005).

A literatura reconhece ainda a relação complexa entre demência, rendimentos e escolaridade. Nesse contexto, o número de anos de estudo tem sido considerado tanto como fator de proteção neuronal quanto como elemento de confusão diagnóstica, já que o desempenho de indivíduos testados com instrumentos de avaliação cognitiva é fortemente influenciado pela escolaridade. Por outro lado, hábitos de vida que podem oferecer riscos de patologias demenciantes são mais freqüentes entre indivíduos com baixa escolaridade, renda e acesso aos bens e serviços (Tombaugh e Mclntyre, 1992; UhImann e Larson, 1991).

Nesse sentido, foi verificado que a renda também influenciou a pontuação do MEEM. Corroborando estes resultados, o estudo desenvolvido na região sudeste verificou maior prevalência de demência em idosos cariocas (15\%) do sexo feminino e que residiam em bairros com menor condição socioeconômica (Veras, 1994).

Além da escolaridade e renda, o próprio envelhecimento é, provavelmente, o principal fator de risco de patologias demenciantes, episódio já conhecido e explorado pela literatura (Tombaugh e Mclntyre, 1992). Desse modo, a idade é uma das variáveis que podem comprometer o desempenho no teste de rastreio da demência, refletindo pior desempenho cognitivo (Brucki et al., 2003; Laks et al., 2003; Diniz et al., 2007). Assim sendo, Brucki et al. (2003), analisando pessoas saudáveis de 16 a 92 anos, identificaram a influência da idade nos escores do MEEM nos grupos mais jovens (16 a 50 anos) e nos mais idosos (65 anos ou mais). Na pesquisa envolvendo idosos atendidos em um ambulatório de São Paulo/SP, foi verificado que o escore total do MEEM apresentou correlação de magnitude moderada com a idade ( $r=-0,41, p<0,001)$, ou seja, quanto mais velho o indivíduo, menor o escore no teste (Almeida, 1998). Além disso, observou-se que os pacientes que receberam o diagnóstico de demência eram mais idosos (73,60 anos, $\mathrm{Cl}=71,44$ a 75,76) que aqueles sem esse diagnóstico (67,29 anos, $\mathrm{Cl}=66,26 \mathrm{a}$ 68,32) ( $t=5,95, \mathrm{p}<0,001$; Cid = 4,22 a 8,40) (Almeida, 1998). Na região sudeste do Brasil, Diniz et al. (2007), avaliando 176 indivíduos com idade entre 65 e 97 anos, constataram que quanto maior a idade, pior é a pontuação no MEEM.

Todavia, no presente estudo não foi observado diferença significativa entre faixa etária e pontuação do MEEM. Este evento pode ser explicado pelo número reduzido da amostra que ao ser dividido em grupos por faixa etária, diminui o poder de mostrar diferenças. Além disso, o percentual de idosos velhos (80 anos ou mais) que participou do estudo foi de apenas 7,5\%. Salienta-se a dificuldade em realizar estudos com pessoas acima de 80 anos, principalmente idosos praticantes de exercícios físicos, uma vez que 
as limitações físicas e outras barreiras pessoais e ambientais podem impedir a inserção desse idoso em programas dessa natureza.

Na população estudada, a prevalência de sintomas depressivos é alta (17,4\%), assemelhando-se às taxas encontradas em outros estudos. No sudeste do Brasil, a prevalência de depressão foi de $25,75 \%$ em idosos cariocas (Veras, 1994) e $24 \%$ em idosas da região nordeste que freqüentavam o Programa Universidade Aberta para a Terceira Idade (Leite et al., 2006).

Quanto à associação entre a GDS e as variáveis sociodemográficas, observou-se que os sujeitos apresentaram medianas iguais quanto a faixa etária, renda e estado civil, ou seja, estas variáveis parecem não influenciar o aparecimento de depressão. Contudo, estudos têm evidenciado associação positiva, como a pesquisa nacional realizada na região nordeste, que detectou associação estatística significativa entre baixa escolaridade e depressão (Leite et al., 2006). Em outro estudo realizado na mesma região, Almeida Filho et al. (2004) constataram que o menor nível educacional estava mais relacionado à presença de transtornos depressivos em mulheres. Cacciatore (1998) também verificou relação entre baixa escolaridade e maior número de idosos deprimidos. Por outro lado, corroborando os dados encontrados, Black e Markides (1998) observaram que o grau de escolaridade apresentava fraca associação com depressão em idosos mexicanos.

Em relação à associação da depressão e do estado civil, no estudo desenvolvido na cidade de Salvador/BA, constatou-se que a condição de não estar casado (solteiro, divorciado ou viúvo) estava associada à menor prevalência de depressão em homens. Para as mulheres, ser solteira era condição associada a menor prevalência de depressão (Almeida Filho et al., 2004). Em contrapartida, Paradela et al. (2005) evidenciaram que a situação conjugal mostrou diferença estatística significativa para o aparecimento de sintomas depressivos, entre os que viviam com um companheiro(a) e aqueles que estavam sozinhos (viúvos e separados). Os autores levantam a hipótese de que os "sozinhos", talvez, queixem-se mais freqüentemente de sintomas depressivos, e que estas queixas estejam associadas à sua solidão (Paradela et al., 2005).

Por sua vez, a renda é um fator associado à menor prevalência de depressão em idosos. No Brasil, o pesquisador Veras (1994) investigou as prevalências de depressão na cidade do Rio de Janeiro, estratificando a população em três estratos, de acordo com o seu nível socioeconômico. As prevalências de depressão variaram de acordo com o grupo estudado, que foi de $22,6 \%$ no distrito com maior poder aquisitivo e $35,1 \%$ no distrito de baixo poder aquisitivo. No entanto, os dados obtidos diferem dessa casuística, ou seja, o nível socioeconômico não influenciou a pontuação da GDS, mesmo evidenciando que 41,1\% dos sujeitos têm renda familiar entre 2 a 3,9 salários, seguido de 21,5\% que recebem até 1,9 salários (Tabela 1).

Em relação à associação entre a depressão e a faixa etária, o estudo desenvolvido no nordeste verificou que grande parte dos idosos deprimidos estava no grupo de idade entre 70 a 79 anos (Leite et al., 2006). Esse dado pode ser justificado pelo estudo de Ramos et al. (1998), no qual se observou que a depressão aumentava com a idade. Por outro lado, os resultados do presente estudo corroboram a pesquisa desenvolvida no centro-oeste que também não encontrou associação positiva entre essas variáveis (Oliveira et al., 2006).

Detectou-se, ainda, que existe relação inversa entre MEEM e GDS, ou seja, baixa pontuação no MEEM pode influenciar o aparecimento de sintomas depressivos. Dessa forma, inferem-se que os sujeitos que apresentaram presença de sintomas depressivos obtiveram pontuação abaixo do normal no MEEM e aqueles com ausência de sintomas depressivos apresentaram pontuação normal no MEEM.

Corroborando esses achados, a pesquisa longitudinal do sul do Brasil com idosos de 80 anos ou mais, aponta que o incremento de sintomas depressivos nos idosos estava associado a pior desempenho no MEEM (Argimon e Stein, 2005). Nesse sentido, idosos deprimidos que apresentam déficits cognitivos proeminentes como parte da síndrome depressiva têm risco aumentado de evoluir para demência, mesmo aqueles que obtêm remissão dos sintomas demenciais após o tratamento bem-sucedido da depressão. Fato observado por Alexopoulos et al. (1993) que verificaram o aumento de cinco vezes no risco de acometimento demencial nos pacientes que apresentavam depressão, em associação com alterações cognitivas, sendo essas reversíveis ou não. Por essa razão, os autores destacam que pacientes geriátricos deprimidos - com ou sem déficits neuropsicológicos evidentes - devem ser avaliados e seguidos com instrumentos de rastreio de demência (Alexopoulos et al., 1993).

\section{CONCLUSÃO}

O presente estudo detectou prevalências significativas para depressão e transtornos cognitivos em idosos iniciantes em um programa de exercício físico. Para esta população, quanto maior a escolaridade e renda, melhor foi o seu desempenho no MEEM, no entanto, não foi observada associação positiva entre depressão e variáveis sociodemográficas. A elevada prevalência de depressão encontrada na população estudada remete à importância de planejamento do programa de exercício físico com a Secretaria Municipal de Saúde, em adotar ações direcionadas à saúde mental dos participantes, em particular, os transtornos mentais relativos à demência e à depressão. 


\section{REFERÊNCIAS}

Alexopoulos G, Meyers BS, Young RC, Mattis S, Kakuma T. The course of geriatric depression with "reversible dementia": a controlled study. Am J Psychiatry. 150:1693-9, 1993.

Almeida OP. Mini exame dos estado mental e o diagnóstico de demência no Brasil. Arq Neuropsiquiatr. 56(3B):605-12, 1998

Almeida OP, Almeida SA. Confiabilidade da versão brasileira da escala de depressão em geriatria (GDS) versão reduzida. Arq. Neuropsiquiatr. 57(2-B): 421-6, 1999.

Almeida OP, Garrido R, Tamai S. Unidade para idosos (UNID) do Departamento de Saúde Mental da Santa Casa de São Paulo: características clínicas de pacientes atendidos em nível ambulatorial. J Bras Psiquiatr. 47:291-6, 1998.

Almeida Filho N. et al. Social inequality and depressive disorders in Bahia, Brazil: interactions of gender, ethnicity and social class. Soc Sci Med. 59:1339-53, 2004.

Argimon IIL, Stein LM. Habilidades cognitivas em indivíduos muito idosos: um estudo longitudinal. Cad. Saúde Pública. 21(1):64-72, 2005

Ávila R, Bottino CMC. Atualização sobre alterações cognitivas em idosos com síndrome depressiva. Rev Bras Psiquiatr. 28(4):316-20, 2006.

Bertolucci PHF, Brucki SMD, Campacci SR, JulianoY. 0 mini-exame do estado mental em uma população geral: impacto da escolaridade. Arq. Neuropsiquiatr. 52:1-7, 1994.

Black SA, Markides KS. Depressive symptoms and mortality in older Mexican americans. Ann Epidemiol. 9:45-52,1998.

Blay SL, Bickel H, Cooper B. Mental illness in cross- national perspective. Results from a Brazilian and German community survey among the elderly. Soc Psychiatr Epidemiol. 26: 245-51,1999.

Brucki SMD, Nitrini R, Caramelli P, Bertolucci PHF, Okamoto IH. Sugestões para o uso do miniexame do estado mental no Brasil. Arq Neuropsiquiatr. 61(3-B):777-81, 2003.

Cacciatore F. Morbidity patterns in aged population in Southern Italy: a survey sampling. Arch Gerontol Geriatric. 26:201-13, 1998.

Camarano AA. Considerações finais. In: Camarano AA, organizador. Muito além dos 60: os novos idosos brasileiros Rio de Janeiro: IPEA, p. 369-82, 1999.

Diniz BSO, Volpe FM, Tavares AR. Nível educacional e idade no desempenho no mini-exame do estado mental em idosos residentes na comunidade. Rev Psiq Clín. 34(1):13-17, 2007.

Fratiglioni L, Jorm AF, Grut M, et al. Predicting dementi from mini-mental state examination in an elderly population: the role of education. J Clin Epidemiol. 48:281-87, 1993.

Herrera E, Caramelli P, Silveira ASB, Nitirni R. Epidemiologic survey of dementia in a communitydwelling Brazilian population. Alzheimer Dis Assoc Disord.16:103-8, 2002.
Ishizaki J, Meguro K, Ambo H, et al. A normative, community-based study of mini-mental state in elderly adults: the effect of age and educational level. J Gerontol B Psychol Sci Soc Sci. 53:359-63, 1998

Jorm AF, Jolley D. The incidence of dementia: a meta-analysis. Neurology. 51:728-33, 1998.

Laks J, Batista EMR, Guilherme ERL, Contino ALB, Faria MEV, Figueira I, et al. 0 mini-exame do estado mental em idosos de uma comunidade. Dados parciais de Santo Antônio de Pádua, Rio de Janeiro. Arq Neuropsiquiatr. 61(3-B):782-85, 2003.

Leite VMM, Carvalho EMF, Barreto KML, Falcão IV. Depressão e envelhecimento: estudo nos participantes do Programa Universidade Aberta à Terceira Idade. Rev Bras Saúde Matern Infant. 6(1):31-8, 2006

Lourenço RA, Veras RP. Mini-exame do estado mental: características psicométricas em idosos ambulatoriais. Rev Saúde Pública. 40(4):712-9, 2006

Oliveira DAAP, Gomes L, Oliveira RF. Prevalência de depressão em idosos que freqüentam centros de convivência. Rev Saúde Pública. 40(4):734-6, 2006.

Paradela EMP, Lourenço RA, Veras RP. Validação da escala de depressão geriátrica em um ambulatório geral. Rev Saúde Pública. 39(6):918-23, 2005.

Penninx BW, Deeg DJ, van Eijk JT, Beekman AT, Guralnik JM. Changes in depression and physical decline in older adults: a longitudinal perspective. J Affect Disorders. 61:1-12, 2000.

Petersen RC, Stevens JC, Ganguli M, Tangalos EG, Cummings JL, Dekosky ST. Practice parameter: early detection of dementia: mild cognitive impairment (an evidence-based review). Report of the Quality Standards Subcommittee of the American Academy of Neurology. Neurology. 56(9):1133-42, 2001.

Ramos L, Toniolo JN, Cendoroglo MS, Garcia JT, Najas MS, Perracini M, et al. Two-year follow-up study od elderly residents in S. Paulo, Brazil: methodology and preliminary results. Rev Saúde Pública. 32(5):397-407,1998.

Reys BN, Bezerra AB, Vilela ALS, Keusen AL, Marinho V, Paula E, et al. Diagnóstico de demência, depressão e psicose em idosos por avaliação cognitiva breve. Rev Assoc Med Bras. 52(6):401-4, 2006

Snowdon J. How high is the prevalence of depression in old age. Rev Bras Psiquiatr. 24 S42-S47, 2002.

Stoppe Júnior A, Louzã Neto MR. Depressão na terceira idade. São Paulo: Lemos, 1998.

Tombaugh TN, Mclntyre NJ. The mini-mental state examination: a comprehensive review. J Am Geriatr Soc. 40:922-35, 1992

Uhlmann RF, Larson EB. Effect of education on the mini-mental state examination as a screening test for dementia. J Am Geriatr Soc. 39:876-80,1991.

Veras RP. País jovem: com cabelos brancos. Rio de Janeiro: Relume Dumará, 1994.

Veras RP, Caldas CP, Dantas SB, Sancho LG, Sicsú B, Motta LB, et al. Avaliação dos gastos com 0 cuidado do idoso com demência. Rev Psiq Clín. 34(1):5-12, 2007.

Yesavage JA et al. Escala de depressão geriátrica (abreviada de Yesavage). In: Freitas EV, Py L, Neri AL, Cançado FAXC, Gorzoni ML, Doll J, organizadores. Tratado de geriatria e gerontologia. Rio de Janeiro: Guanabara Koogan, p. 1154, 2002 